\title{
Administrative and syndromic surveillance data can enhance public health surveillance
}

\author{
Tara C. Anderson*, Hussain Yusuf, Amanda McCarthy, Katrina Trivers, Peter Hicks, \\ Michael Coletta and Violanda Grigorescu
}

Division of Health Informatics and Surveillance, Center for Surveillance, Epidemiology, and Laboratory Services, Office of Public Health Scientific Services, Centers for Disease Control and Prevention, Atlanta, GA, USA

\section{Objective}

This roundtable will address how multiple data sources, including administrative and syndromic surveillance data, can enhance public health surveillance activities at the local, state, regional, and national levels. Provisional findings from three studies will be presented to promote discussion about the complementary uses, strengths and limitations, and value of these data sources to address public health priorities and surveillance strategies.

\section{Introduction}

Healthcare data, including emergency department (ED) and outpatient health visit data, are potentially useful to the public health community for multiple purposes, including programmatic and surveillance activities. These data are collected through several mechanisms, including administrative data sources [e.g., MarketScan claims data ${ }^{1}$; American Hospital Association (AHA) data $\left.{ }^{2}\right]$ and public health surveillance programs [e.g., the National Syndromic Surveillance Program (NSSP) ${ }^{3}$ ]. Administrative data typically become available months to years after healthcare encounters; however, data collected through NSSP provide near real time information not otherwise available to public health. To date, 46 state and 16 local health departments participate in NSSP, and the estimated national percentage of ED visits covered by the NSSP BioSense platform is $54 \%$. NSSP's new data visualization tool, ESSENCE, also includes additional types of healthcare visit (e.g., urgent care) data. Although NSSP is designed to support situational awareness and emergency response, potential expanded use of data collected through NSSP (i.e., by additional public health programs) would promote the utility, value, and long-term sustainability of NSSP and enhance surveillance at the local, state, regional, and national levels. On the other hand, studies using administrative data may help public health programs better understand how NSSP data could enhance their surveillance activities. Such studies could also inform the collection and utilization of data reported to NSSP.

\section{Keywords}

Administrative data; Syndromic surveillance; Public health surveillance

\section{References}

${ }^{1}$ Truven Health Analytics. MarketScan Research Databases. Truven Health Analytics website. http://truvenhealth.com/your-healthcarefocus/analytic-research/marketscan-research-databases. Accessed September 9, 2016.

${ }^{2}$ American Hospital Association (AHA). AHA Data and Directories. AHA website. http://www.aha.org/research/rc/stat-studies/data-anddirectories.shtml. Accessed September 9, 2016.

${ }^{3}$ Centers for Disease Control and Prevention (CDC). National Syndromic Surveillance Program. CDC website. http://www.cdc.gov/nssp/. Last updated July 29, 2016. Accessed September 9, 2016.

\author{
*Tara C. Anderson \\ E-mail: TCAnderson1@cdc.gov
}

